\title{
CONCERNING SUSPENSION SPHERES
}

\author{
RONALD H. ROSEN
}

1. Introduction. It was first pointed out by $M$. Hirsch that the suspension of a smooth closed 4-manifold $M^{4}$ having the homotopy type of $S^{4}$, is topologically a 5-sphere [6]. P. Harley [5] has shown that recent developments in the theory of manifolds allow one to strengthen Hirsch's result by dropping the condition that $M^{4}$ be smooth. Independently of Harley we have formulated a more general suspension theorem; however, it is easy to see that the basic ideas of our proof are quite similar to his proof. By use of examples and applications we shall, therefore, demonstrate that our result is a real nonvacuous generalization of his.

Our main result is Theorem 4 . Let $Z$ be a compact metric space which is 1-connected and has the homology of $S^{n}$. Suppose that $Z \times R^{1}$ is an unbounded $(n+1)$-manifold. If $n \geqq 4$ and $Z$ contains a topological $n$-cell, the suspension of $Z$ is a topological $(n+1)$-sphere.

It should be remembered that, following Bing's example [2], there have been numerous constructions of nonmanifolds which suspend to spheres.

With minor modifications we shall use notation adopted by us in [14], [15], [16]. All spaces are assumed to be $T_{2}$. A manifold shall be considered to have no boundary unless it is explicitly referred to as a manifold with boundary. $X * Y$ shall be the join of $X$ and $Y$. OC $(Y)$ will stand for the open cone $C(Y)-Y$.

Following the terminology of M. Brown [4], we shall say that a closed pair $(X, A)$ is collared (bicollared) if $A$ has a closed neighborhood $Y$ equivalent to $A \times I(A \times[-1,1])$ such that $A$ corresponds to $A \times\{0\}$ and $\mathrm{Bd} Y$ corresponds to $A \times\{1\}(A \times\{-1,1\})$.

If $(X, A)$ and $(Y, B)$ are closed pairs and $f$ is a homeomorphism of $A$ on $B$, then $(X, A) \#(Y, B)(f)$ will denote the adjunction space formed from the disjoint union of $X$ and $Y$ via identification by $f$. When there seems to be little possibility of confusion we may use $(X, A) \#(Y, B)$ or even $X \# Y$ for this type of connected sum. If $\left(X^{\prime}, A^{\prime}\right)$ is a disjoint copy of $(X, A)$, then $(X, A) \#\left(X^{\prime}, A^{\prime}\right)$ will be called the double of $(X, A)$ and represented by $2(X, A)$ or possibly $2 X$.

\section{Some properties of collared pairs.}

Lemma 1. Let $(X, A)$ be a closed metric pair such that $A$ has an open collar (bicollar) neighborhood in $X$. Then $(X, A)$ is collared (bicollared).

Received by the editors December 5, 1968. 
Proof. This is a simple application of Brown's construction of spindle neighborhoods ( $\$ 3$ of $[4]$ ) and his observation that they form a neighborhood base for $A \times 0$ in $A \times[0,1)$.

Lemma 2. Suppose $(X, A)$ is a collared pair. Let $X^{\prime}$ be the subset $X \times\{0\} \cup A \times I \cup X \times\{1\}$ of $X \times I$.

(A) $X^{\prime} \approx 2(X, A)$.

(B) If $X$ is metric then $\left(X \times I, X^{\prime}\right)$ is a collared pair.

Proof. (A) This is an elementary exercise involving homeomorphisms of closed intervals.

(B) Choose a neighborhood $N$ of $A$ in $X$ with $(N, A) \approx(A \times I, A \times 0)$. Each point of $X^{\prime}-(A \times I)$ obviously has an open collar neighborhood in $X \times I$. On the other hand $N^{\prime}=N \times I$ is a neighborhood of $A \times I$ in $X \times I$. Since $N^{\prime}$ is equivalent to $A \times I \times I, X^{\prime}$ is locally collared in $X \times I$. The conclusion follows from Theorem 1 of [4] and Lemma 1 above.

Lemma 3. Let $(Y, B)$ be a compact subpair of $S^{n}$. Suppose $B \subseteq$ Int $Y$ and $f$ is a homeomorphism of $(\mathrm{Bd} Y) \times[0,1)$ onto $Y-B$. Then the following statements are equivalent.

(A) $B$ is cellular.

(B) $S(\operatorname{Bd} Y) \approx S^{n}$.

(C) Int $Y \approx R^{n}$.

Proof. From dimension considerations and invariance of domain it follows that $f(\mathrm{Bd} Y \times 0)=\mathrm{Bd} Y$. Let $Y_{t}=f(\mathrm{Bd} Y \times[t, 1)) \cup B$, $0 \leqq t<1$. It is clear that $Y_{t} \approx Y$ and $B=\lim _{t \rightarrow 1} Y_{t}$. If $K \subseteq Y$ let $K_{t}$ be its image in $Y_{t}$.

$(\mathrm{A}) \Rightarrow(\mathrm{B})$. Since $B$ is cellular there is an embedding $g$ of $C(\operatorname{Bd} Y)$ in $S^{n}$ in which $g(\mathrm{OC}(\mathrm{Bd} Y))$ is a neighborhood of the cone vertex. By Theorem 4 of [14], $S(\operatorname{Bd} Y) \approx S^{n}$.

$(\mathrm{B}) \Rightarrow(\mathrm{C})$. Bd $Y_{1 / 2}$ is bicollared in $S^{n}$. Applying Lemma 1 of [16], we note that $\mathrm{Bd} Y_{1 / 2}$ is flat in $S^{n}$ and Int $Y \approx \operatorname{Int} Y_{1 / 2} \approx R^{n}$.

$(\mathrm{C}) \Rightarrow(\mathrm{A})$. Let $D$ be a topological $n$-cell in Int $Y$ so that $B \subseteq$ Int $D$. Thus $B=\lim _{t \rightarrow 1} D_{t}$, and $B$ is cellular.

REMARK. This lemma might be compared to some previously used results, e.g. Lemma 7 of [15] and Theorem 6 of [14].

As an application of some of these notions, we give a simple proof of a fact which has appeared in the literature in various less general guises. We have used it to verify the corollaries to Theorems 1 and 2 in [15]. One might also refer to Proposition 5.5 of [9]. 
Theorem 1. Let $(X, A)$ be a collared pair. Then $2(X, A) \approx S^{n}$ iff $X \times I \approx I^{n+1}$ and $(X-A) \times(0,1)$ is a manifold.

Proof. "if." It follows that $\partial I^{n+1}=\partial(X \times I)=X \times 0 \cup A \times I \cup X \times 1$. We then apply Lemma 2 (A).

"only if." Suppose $2(X, A)=(X, A) \#\left(X^{\prime}, A^{\prime}\right)=S^{n}$. This implies that $X-A$ is open in $S^{n}$. Let $p \in \operatorname{Int} X^{\prime}$. We may identify $S^{n}-p$ with $R^{n}$. Consider the subset $X \times I \subseteq R^{n} \times R^{1}=R^{n+1}$. By Lemma $2(X \times I$, $\operatorname{Bd}(X \times I))$ is a collared pair and $\operatorname{Bd}(X \times I) \approx 2(X, A) \approx S^{n}$. By [3], $X \times I \approx I^{n+1}$.

The next result is used in our suspension theorem. Although it may be avoided there by appealing to properties of generalized manifolds, we include it because its proof is quite elementary.

Theorem 2. Let $(X, A)$ be a normal bicollared pair. Suppose $X$ is connected and locally 0-connected, and $A$ is 0-connected. If the singular integral group $H_{1}(X)$ has rank zero, $A$ bounds exactly two complementary domains in $X$.

Proof. Let $Y$ be a closed neighborhood of $A$ which we can identify with $A \times[-1,1]$, so that $A$ corresponds to $A \times 0$ and Bd $Y$ corresponds to $A \times\{-1,1\}$. Let Int $Y=N$ and $Z=X-N$. With no loss of generality we may assume that $(Z, \mathrm{Bd} Z)=(Z, \mathrm{Bd} Y)$ is a collared pair. This allows us to conclude that if $\mathrm{Bd} Y$ lies in one component of $X-A$, then $\mathrm{Bd} Y$ lies in one component of $Z$. We show that this is impossible by a unicoherence type of argument. Since the classical results in this direction [21] and [22] are stated for Peano spaces, we first sketch this part of the proof.

From our assumptions there is an arc $J$ in $Z$ which meets Bd $Y$ in its endpoints $p_{i}=\left(x_{i}, i\right) \in A \times\{i\}, i= \pm 1$. Let $B=J \cup \mathrm{Bd} Y$ and $r: B \rightarrow J$ be the map which is constant on each component of $\mathrm{Bd} Y$ and the identity on $J$. This map may be extended over $Z$, for $(Z, B)$ is a closed normal pair.

There is an embedding $f:[-1,1] \rightarrow A$ with $f(i)=x_{i}, i= \pm 1$. Consequently $K=\{(f(t), t) \mid-1 \leqq t \leqq 1\}$ is an arc in $Y$ which meets Bd $Y$ in its endpoints $p_{-1}$ and $p_{1}$. Mirroring the previous argument there is a retraction $r^{\prime}$ of $Y$ on $K$ which is constant on the components of Bd $Y$. Hence $r \cup r^{\prime}$ is a retraction of $X$ onto the simple closed curve $J \cup K$. This would mean that the infinite cyclic group $H_{1}(J \cup K)$ is a summand of $H_{1}(X)$, which is impossible.

Accordingly the connected sets $A \times[-1,0)$ and $A \times(0,1]$ lie in distinct components of $X-A$. Since $N$ is a neighborhood of $A$ which meets only these two complementary domains, we are done. 
3. Suspension theorems. We call $X$ a suspension $n$-sphere (or suspension $n$-cell) if its suspension $S^{0} * X=S(X)$ is topologically the same as $S^{n+1}$ (or $\left.I^{n+1}\right)$. The integer $n$ may be omitted if the dimension is evident.

Lemma 4. Let $(X, A)$ be a bicollared pair in which $A$ is a suspension sphere. Then $X$ is a suspension $n$-sphere iff $A$ bounds two suspension n-cells.

Proof. In either case, from Theorem $2, A$ bounds two closed domains $D_{1}$ and $D_{2}$. Evidently $\operatorname{dim} X=n$ and $\operatorname{dim} A=n-1$.

"if." Then $S(X)$ is the union of the cells $S\left(D_{i}\right)$ which meet on their common boundary $S(A) \approx S^{n}$.

"only if." If $n<3$, the proof is trivial. If $n \geqq 3$, the $n$-sphere $S(A)$ is bicollared in the $(n+1)$-sphere $S(X)$ except possibly at the suspension vertices. According to Kirby [7], $S(A)$ is flat. This implies that $S\left(D_{i}\right)$ is an $(n+1)$-cell.

Theorem 3. Let $(X, A)$ be a collared compact metric pair. Suppose $A$ is a suspension sphere, $X$ is $\infty$-connected and $(X-A) \times R^{1}$ is an $(n+1)$-manifold. If $n \geqq 4, X$ is a suspension $n$-cell.

Proof. Again from dimension theory $\operatorname{dim} X=n$ and $\operatorname{dim} A=n-1$. Thus $A$ is a suspension $(n-1)$-sphere. Accordingly $X$ and $A$ are ANR's so that $X$ is contractible and $A$ has the homotopy type of $S^{n-1}$. Let $Y=X \times I$ and $X^{\prime}=X \times\{0\} \cup A \times I \cup X \times\{1\}$. By Lemma 2 , $\left(Y, X^{\prime}\right)$ is a collared pair and we may see that $Y-X^{\prime}=(X-A)$ $X(0,1)$ is an $(n+1)$-manifold. Using Van Kampen's Theorem and the hypothesis $n \geqq 4$, it is evident that $X^{\prime}$ is $[(n+1) / 2]$-connected. Since $A$ is collared in $X, X-A$ and thus $Y$ and $Y-X^{\prime}$ are all contractible. Consider the pair $\left(2 Y, X^{\prime}\right)$. Since it is bicollared, $2 Y$ is a closed $(n+1)$-manifold. $2 Y$ is $[(n+1) / 2]$-connected and $n+1 \geqq 5$. Therefore by Theorem 7 of [10], $2 Y \approx S^{n+1}$. Int $Y=Y-X^{\prime}$ is contractible, open in $S^{n+1}$ and simply connected at infinity. From Theorem 4 of [18], we may conclude that Int $Y \approx R^{n+1}$. Again inasmuch as $\left(Y, X^{\prime}\right)$ is collared, it is a consequence of Lemma 3 that $S^{n+1}$ $\approx S\left(X^{\prime}\right) \approx S(2(X, A))$. Finally by Lemma $4 S(X) \approx I^{n+1}$.

Theorem 4 (Alternative statement). Let $(Z, A)$ be a bicollared compact metric pair. Suppose $A$ is a suspension sphere, $Z \times R^{1}$ is an $(n+1)$-manifold and $Z$ is a 1-connected homology $n$-sphere with $n \geqq 4$. Then $S(Z) \approx S^{n+1}$.

REMARK. First let us note the equivalence of this statement with that given in the introduction. If $Z$ has a subset $U$ homeomorphic to 
$R^{n}$, we note that $U \times R^{1}$ is open in $Z \times R^{1}$ by invariance of domain, so $U$ is open in $Z$. Select a bicollared $(n-1)$-sphere in this euclidean neighborhood. On the other hand if $A$ is a bicollared suspension sphere in $Z, \operatorname{dim} A=n-1$. Since $A \times R^{1} \approx S^{n-1} \times R^{1}, Z$ contains an $n$-euclidean open subset.

Proof. From Theorem 2, we know that $A$ bounds exactly two closed domains in $Z$. Alternatively one may use Theorem 6 of [11] and Theorem 3.1 on p. 294 of [19].

Let $X$ be one of the closed domains bounded by $A$. Since $(X, A)$ is collared and $A$ is bicollared, it is easily established that $X$ is an ANR. It is 1-connected and homologically acyclic, and therefore contractible. The pair $(X, A)$ satisfies the hypotheses of Theorem 3 , so $S(X) \approx I^{n+1}$. By Lemma $4, S(Z) \approx S^{n+1}$.

COROllary. Let $K$ be an $n$-polyhedron so that $K \times R^{1}$ is a manifold. If $K$ is a homotopy $n$-sphere and $n \geqq 4, S(K) \approx S^{n+1}$.

Proof. $K$ is $n$-euclidean on the interior of one of its $n$-simplexes. Theorems 3 and 4 generalize Harley's Theorems 2 and 1, respectively.

\section{Some applications.}

Theorem 5. Let $\left(X_{i}, A_{i}\right), i=1,2$, be collared compact metric pairs. Suppose $X_{i}$ is contractible and $A_{i}$ is homologically [n/2]-connected. Assume $n \geqq 4, f: A_{1} \approx A_{2}$ and $\left(X_{i}-A_{i}\right) \times R^{1}$ is an $(n+1)$-manifold. Then $Z=\left(X_{1}, A_{1}\right) \#\left(X_{2}, A_{2}\right)(f)$ is a suspension sphere if it contains an $n$-cell.

Proof. Clearly $X_{i}$ and $A_{i}$ are ANR's. By Van Kampen's Theorem, $\pi_{1}(Z)$ is trivial. From the Mayer-Vietoris sequence, $H_{k}\left(A_{1}\right) \rightarrow H_{k}\left(X_{1}\right)$ $\oplus H_{k}\left(X_{2}\right) \rightarrow H_{k}(Z) \rightarrow H_{k-1}\left(A_{1}\right)$, we note that $Z$ is homologically $[n+1 / 2]$-connected. $Z \times R^{1}$ is an orientable $(n+1)$-manifold. We may now use duality to show that $Z$ is a homology $n$-sphere. By Theorem $4, S(Z) \approx S^{n+1}$.

We recall that nonmanifold suspension $n$-spheres and $n$-cells have been constructed by Bing [2], Rosen [12], [13, \$1, p. 565], Kwun [8] and Andrews-Curtis [1]. On the other hand for each $n \geqq 4$, there is a contractible manifold $M^{n}$ which has the properties that, $\partial M^{n}$ is not 1 -connected but $M^{n} \times I$ is an $(n+1)$-cell [20]. Using these types of examples, Theorem 5 indicates that one may take various kinds of connected sums of contractible nonmanifolds bounded by collared nonsimply connected homology $(n-1)$-spheres, and still produce suspension $n$-spheres.

THEOREM 6. Let $G$ be a finite collection of disjoint compact subsets of 
$S^{n}$. Suppose each $C \in G$ is locally 0-connected and homologically $n$-connected. Then if $n \geqq 4$ and $S^{n} / C \times R^{1}$ is an $(n+1)$-manifold for each $C \in G$, $S^{n} / G$ is a suspension sphere.

Proof. By [17], the induced map $\pi_{1}\left(S^{n}\right) \rightarrow \pi_{1}\left(S^{n} / G\right)$ is epic so that $S^{n} / G$ is 1 -connected. By the Begle-Vietoris mapping theorem $H_{i}\left(S^{n}\right)$ $\approx H_{i}\left(S^{n} / G\right)$ for $0 \leqq i \leqq n . S^{n} / G$ is $n$-dimensional so that it is a homology $n$-sphere. From the conditions $S^{n}-\cup\{C \mid C \in G\} \neq \varnothing$, so that $S^{n} / G$ is $n$-euclidean at some of its points.

If $C \in G$ let $U$ be one of its open neighborhoods which meets no other element of $G$. Accordingly $U / G=U / C$ and $U / C \times R^{1}$ is an $(n+1)$-manifold. This means that $S^{n} / G \times R^{1}$ is an $(n+1)$-manifold. Now the conclusion follows from an application of Theorem 4.

Lemma 5. Let $G$ be a collection of disjoint arcs in $S^{n}$. Then $S^{n} / G$ is a suspension sphere.

Proof. For $n \geqq 4$ this is a "corollary" to [1] and Theorem 6. As was correctly pointed out by Kwun and Raymond in $\$ 5$, p. 117 of [17], however, this is true for all $n$ by examination of the method of proof in $[1]$.

THeorem 7. Let $M$ be a triangulated $n$-manifold and $T$ be a subpolyhedron which is a tree. Consider a barrel neighborhood $B$ of $T$ in $M$.

If $n=4, \mathrm{Bd} B$ is a suspension 3-sphere and $T$ is cellular in $M$.

If $T$ is an arc and $n \geqq 5, S(\mathrm{Bd} B)$ is a suspension $n$-sphere.

Proof. Examination of the proofs of Theorems 1 and 2 of [15] reveals that an inductive procedure is carried out based on the number of vertices of $T . B$ is equivalent to the union of barrel neighborhoods $B_{0}$ and $B_{1}$ of proper subtrees. Furthermore $B_{0} \cap B_{1}=C(A)$ $\subseteq \mathrm{Bd} B_{0} \cap \mathrm{Bd} B_{1}$ where $A$ is a bicollared homology $(n-2)$-sphere in $\mathrm{Bd} B_{i}, i=0,1$. Thus $\mathrm{Bd} B=\left(B_{0}-\mathrm{OC}(A), A\right) \#\left(B_{1}-\mathrm{OC}(A), A\right)$. If $n=4, A$ is actually a 2 -sphere and $\mathrm{Bd} B$ is a 3 -manifold. From Lemma 4 , by induction it follows that $\mathrm{Bd} B$ is a suspension 3 -sphere.

According to [15], $2 B \approx S^{n}$ and $B$ is a mapping cylinder from $B d B$ over $T$. For $n=4$, Lemma 3 now implies that $T$ is cellular in $B$.

If $n \geqq 5$ and $T \approx I$, let $S^{n}=B \# B^{\prime}$ and $T^{\prime}$, the disjoint copy of $T$ in $B^{\prime}$. By Lemma $5, S^{n} /\left\{T, T^{\prime}\right\}$ is a suspension $n$-sphere, and it is obviously homeomorphic to $S(\mathrm{Bd} B)$.

\section{BIBLIOGRAPHY}

1. J. Andrews and M. Curtis, $n$-space modulo an arc, Ann. of Math. (2) 75 (1962), $1-7$.

2. R. H. Bing, The cartesian product of a certain nonmanifold and a line is $E^{4}$, Ann. 
of Math. (2) 70 (1959), 399-412.

3. M. Brown, $A$ proof of the generalized Schoenflies theorem, Bull. Amer. Math. Soc. 66 (1960), 74-76.

4. - Locally flat imbeddings of topological manifolds, Ann. of Math. (2) 75 (1962), 331-341.

5. P. W. Harley, On suspending homotopy spheres, Proc. Amer. Math. Soc. 19 (1968), 1123-1124.

6. M. Hirsch, "On homotopy spheres of low dimension" in Differential and combinatorial topology, Princeton Univ. Press, Princeton, N. J., 1965.

7. R. Kirby, On the set of non-locally flat points of a submanifold of codimension one, Ann. of Math. (2) 88 (1968), 281-290.

8. K. Kwun, Factors of N-space, Michigan Math. J. 9 (1962), 207-211.

9. K. Kwun and F. Raymond, Manifolds which are joins, Trans. Amer. Math. Soc. 111 (1964), 108-120.

10. M. H. A. Newman, The engulfing theorem for topological manifolds, Ann. of Math. (2) 84 (1966), 555-571.

11. F. Raymond, Separation and union theorems for generalized manifolds with boundary, Michigan Math. J. 7 (1960), 7-21.

12. R. Rosen, $E^{4}$ is the cartesian product of a totally non-euclidean space and $E^{1}$, Ann. of Math. (2) 73 (1961), 349-361.

13. - Examples of non-orthogonal involutions of euclidean spaces, Ann. of Math. (2) 78 (1963), 560-566.

14. - Stellar neighborhoods in polyhedral manifolds, Proc. Amer. Math. Soc. 14 (1963), 401-406.

15. - Polyhedral neighborhoods in triangulated manifolds, Bull. Amer. Math. Soc. 69 (1963), 359-361.

16. — The five dimensional polyhedral Schoenflies theorem, Bull. Amer. Math. Soc. 70 (1964), 511-516.

17. S. Smale, $A$ Vietoris mapping theorem for homotopy, Proc. Amer. Math. Soc. 8 (1957), 604-610.

18. J. Stallings, The piecewise-linear structure of Euclidean space, Proc. Cambridge Philos. Soc. 58 (3) (1962), 471-488.

19. R. L. Wilder, Topology of manifolds, Amer. Math. Soc. Colloq. Publ., vol. 32, Amer. Math. Soc., Providence, R. I., 1963.

20. M. Curtis, Cartesian products with intervals, Proc. Amer. Math. Soc. 12 (1961), 819-820.

21. K. Borsuk, Ueber die Abbildungen der metrischen kompakten Raüme auf die Kreislinie, Fund. Math. 20 (1933), $230 \mathrm{ff}$.

22. E. Čech, Sur les continus peaniens unicohérents, Fund. Math. 20 (1933), $232 \mathrm{ff}$.

The University of Michigan 